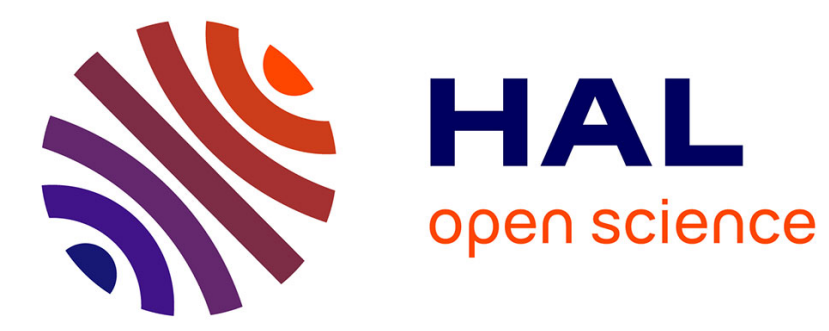

\title{
Titin-induced force enhancement and force depression: A 'sticky-spring' mechanism in muscle contractions?
}

\author{
Christian Rode, Tobias Siebert, Reinhard Blickhan
}

\section{To cite this version:}

Christian Rode, Tobias Siebert, Reinhard Blickhan. Titin-induced force enhancement and force depression: A 'sticky-spring' mechanism in muscle contractions?. Journal of Theoretical Biology, 2009, 259 (2), pp.350. 10.1016/j.jtbi.2009.03.015 . hal-00554591

\section{HAL Id: hal-00554591 \\ https://hal.science/hal-00554591}

Submitted on 11 Jan 2011

HAL is a multi-disciplinary open access archive for the deposit and dissemination of scientific research documents, whether they are published or not. The documents may come from teaching and research institutions in France or abroad, or from public or private research centers.
L'archive ouverte pluridisciplinaire HAL, est destinée au dépôt et à la diffusion de documents scientifiques de niveau recherche, publiés ou non, émanant des établissements d'enseignement et de recherche français ou étrangers, des laboratoires publics ou privés. 


\section{Author's Accepted Manuscript}

Titin-induced force enhancement and force depression: A 'sticky-spring' mechanism in muscle contractions?

Christian Rode, Tobias Siebert, Reinhard Blickhan

PII:

S0022-5193(09)00114-3

DOI: doi:10.1016/j.jtbi.2009.03.015

Reference: YJTBI 5490

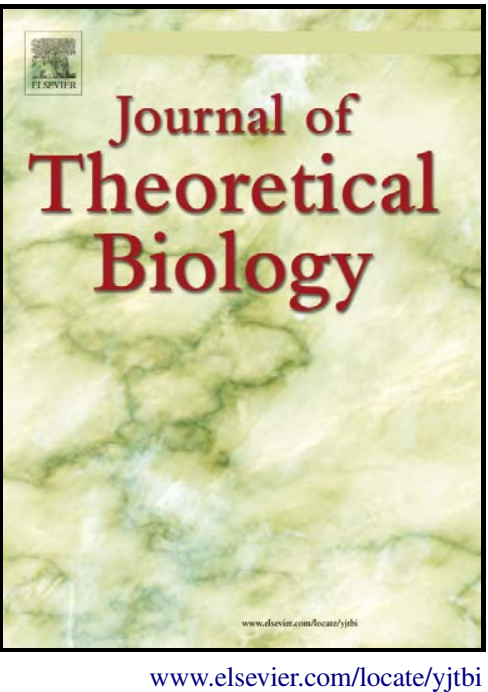

To appear in: $\quad$ Journal of Theoretical Biology

Received date: 12 December 2008

Revised date: 2 March 2009

Accepted date: $\quad 4$ March 2009

Cite this article as: Christian Rode, Tobias Siebert and Reinhard Blickhan, Titin-induced force enhancement and force depression: A 'sticky-spring' mechanism in muscle contractions?, Journal of Theoretical Biology (2009), doi:10.1016/j.jtbi.2009.03.015

This is a PDF file of an unedited manuscript that has been accepted for publication. As a service to our customers we are providing this early version of the manuscript. The manuscript will undergo copyediting, typesetting, and review of the resulting galley proof before it is published in its final citable form. Please note that during the production process errors may be discovered which could affect the content, and all legal disclaimers that apply to the journal pertain. 


\title{
Titin-induced force enhancement and force depression:
}

\section{A 'sticky-spring' mechanism in muscle contractions?}

\author{
Christian Rode*, Tobias Siebert and Reinhard Blickhan \\ Institute of Motion Science, Friedrich Schiller University, \\ Seidelstr. 20, 07749 Jena, Germany \\ *Corresponding author, christian.rode@uni-jena.de, \\ Tel.: 00493641 945704, Fax: 00493641945702
}

The sliding filament and crossbridge theories do not suffice to explain a number of muscle experiments. For example, from the entire muscle to myofibrils, predictions of these theories were shown to underestimate the force output during and after active tissue stretch. The converse applies to active tissue shortening.

In addition to the crossbridge cycle, we propose that another molecular mechanism is effective in sarcomere force generation. We suggest that, when due to activation, myosin binding sites are available on actin, the giant protein titin's PEVK region attaches itself to the actin filament at those sites. As a result, the molecular spring length is dramatically reduced. This leads to increased passive force when the sarcomere is stretched and to decreased or even negative passive force when the sarcomere shortens. Moreover, during shortening, the proposed mechanism interferes with active-force production by inhibiting crossbridges.

Incorporation of a simple 'sticky-spring' mechanism model into a Hill-type model of sarcomere dynamics offers explanations for several force-enhancement and force-depression effects. For example, the increase of the sarcomere force compared to the force predicted solely by the sliding filament and crossbridge theories depends on the stretch amplitude and on the working range. The same 
applies to the decrease of sarcomere force during and after shortening. Using only

27 literature data for its parameterization, the model predicts forces similar to experimental results.

Key words: molecular mechanism, model, stretch, shortening, sarcomere, myofilament

31 interaction

\section{Introduction}

From whole muscle to myofibrils, the steady-state force after an active stretch can exceed the force measured in an isometric contraction at the same final length (Abbott and Aubert, 1952; Edman et al., 1982) and can even exceed the maximum isometric force (Herzog et al., 2008; Lee and Herzog, 2008). While the first result might be explained with non-uniform sarcomere lengthening (Morgan, 1990), the latter cannot be explained solely within the framework of the sliding filament (Huxley and Niedergerke, 1954; Huxley and Hanson, 1954) and the crossbridge theories (Huxley, 1957). The physiological mechanism(s) underlying enhanced forces have not been understood to date (see Herzog et al., 2008 for a review). However, it has been suggested that residual force enhancement (the force difference between the two measured steady-state forces) can be separated into active and passive parts (Herzog et al., 2006) related to the crossbridge mechanism and to titin, respectively (Herzog et al., 2008). In contrast, Pinniger et al. (2006) provided evidence suggesting that force enhancement during and after stretch results from a mechanism independent of the crossbridges and may be related to titin.

Stretching active fibers from the same initial length (throughout the manuscript

50 initial refers to the length at which activation occurs) with different constant velocities,

51 Edman et al. (1982) found constant stiffness greater than zero after a short transient 
52 phase, suggesting a spring-like characteristic (elongation-dependent) of force

53 enhancement. Moreover, muscles stretched on the descending limb of the active force-

54 length relationship exceeded the isometric force at the stretched length by a larger

55 amount than muscles stretched the same distance on the ascending limb (Morgan et al.,

56 2000; Peterson et al., 2004). These effects are hard to explain with enhanced crossbridge

57 forces. Not only would the crossbridge have to exert force rising with stretch amplitude,

58 but it would also have to 'know' about the initial filament overlap. This substantiates

59 the presence of a force-producing mechanism independent of the crossbridge force-

60 generation producing increasing forces with increasing initial sarcomere length.

61 Another reported phenomenon of muscular contraction is the decreased steady-state

62 force after active muscle (e.g. Abbott and Aubert, 1952) or fiber (e.g. Edman, 1975,

63 1996; Edman et al., 1993; Sugi and Tsuchiya, 1988) shortening compared to the force

64 measured in an isometric contraction at the same final length. Residual force depression

65 (force difference between these two measured steady state forces) was found to be

66 proportional to the shortening distance (Marechal and Plaghki, 1979; Edman, 1975;

67 Herzog and Leonard, 1997). Mechanisms proposed in this context include stress-

68 induced inhibition of crossbridges (Marechal and Plaghki, 1979).

69 Physiological explanations, as well as most phenomenological descriptions, deal

70 with either force enhancement (the increase in experimental force compared to the force

71 expected from valid sliding filament and crossbridge theories; e.g. Morgan et al., 1982)

72 or with force depression (the converse of the above; e.g. Marechal and Plaghki, 1979;

73 Meijer et al., 1998). Forcinito et al. (1998) showed that a physiologically non-motivated

74 recruitable elastic rack conceptually explains force enhancement and force depression

75 effects. However, in contradiction to experimental results (Herzog and Leonard, 2000),

76 such modeling predicts commutativity of shortening-stretch vs. stretch-shortening

77 experiments with respect to the subsequent steady-state muscle force. 
In this study, we propose and model a physiological mechanism related to titin

79 (connectin) to describe both force-enhancement and force-depression effects. The giant protein titin is a structural part of myosin with a free part acting as a molecular spring connecting myosin to the Z-disc near the actin filament (Linke et al., 1998; Prado et al. 2005). This molecular spring consists of three morphologically distinct regions: 1) the proximal Immunoglobulin (Ig) region connecting to the Z-disc, 2) the adjacent PEVK region (rich in proline $(\mathrm{P})$, glutamic acid $(\mathrm{E})$, valine $(\mathrm{V})$ and lysine $(\mathrm{K})$ ), and 3$)$ the distal Ig region attached to myosin. Interaction with actin seems possible when calcium is present (Kellermayer and Granzier, 1996) and the PEVK region can attach to the actin filament (Bianco et al., 2007). Such attachments may occur at myosin binding sites on actin (Niederlander et al., 2004). Here, we assume that the PEVK region attaches itself to the myosin binding sites on actin during activation (Fig. 1, top), thereby altering titin's behavior during subsequent length changes compared to passive

91 length changes. This 'sticky-spring' mechanism might induce considerable forces during and after sarcomere stretch, as well as during and after sarcomere shortening.

By dramatically reducing titin's free spring length in the active state (i.e. when

94 calcium concentration is high), the 'sticky-spring' mechanism may lead to 1) increased passive half-sarcomere force during and after active stretch and 2) decreased or even negative passive half-sarcomere force during and after active shortening. Moreover,

97 during shortening, the proposed mechanism may interfere with active-force production

98 by inhibiting crossbridges. Resultant, the mechanism might offer an explanation for several force-enhancement and force-depression effects including the noncommutativity (Herzog and Leonard, 2000) of those effects. 
104 We restricted our modeling to the half-sarcomere as the force-producing unit of the 105 muscle (Fig. 1) and neglected elasticity in series to the crossbridges such as elasticity of 106 the actin and myosin filaments. In addition, we normalized forces to the maximum 107 isometric active force $F_{[i m]}$ (throughout the manuscript, values subscripted with brackets 108 were constant for at least one experiment) produced at optimal thin (actin) and thick 109 (myosin) filament overlap and assumed that all forces would act along the myofilament 110 axis.

111 The normalized half-sarcomere force $f_{h s}$ equaled the sum of an active (crossbridges) 112 component $f_{\text {act }}$ and a passive (titin) component $f_{\text {pass }}$ :

$114 \quad f_{h s}=F_{h s} / F_{[i m]}=f_{a c t}+f_{p a s s}$.

116 A common Hill-type separation approach matching the sliding filament and crossbridge

117 theories defined the active force:

118

$$
f_{a c t}=F_{a c t} / F_{[i m]}=f_{v}\left(v_{h s}\right) \cdot f_{l}\left(l_{h s}, l_{h s[0]}\right) .
$$

121 The $f_{v}$ is a factor due to the force-velocity (Hill, 1938; Abbot and Aubert, 1952) relation

122 (shortening: $f_{v}<1$; stretch: $f_{v}>1$ ) and depends on the half-sarcomere velocity $v_{h s}$. The $f_{l}$ 123 is a factor due to the force-length (Gordon et al., 1966) relation $\left(f_{l} \leq 1\right)$. According to the 124 sliding filament theory, $f_{l}$ depends on the number of effective crossbridges. Moreover, 125 on the steep part of the ascending limb of the active force-length relationship, it also 126 assumedly depends on counteracting compressive forces. Following the proposed 127 mechanism, however, $f_{l}$ can be decreased due to inhibited crossbridges; it not only 
128 depends on the half-sarcomere length $l_{h s}$, but also on the initial half-sarcomere length

$129 l_{h s[0]}($ please see appendix A).

130 We modeled titin's molecular spring region as an elastic spring. In the passive state

131 (calcium concentration is low, i.e. the sarcomere is deactivated), $f_{\text {pass }}$ is a function of $l_{h s}$.

132 In the active state (calcium concentration is high, i.e. the sarcomere is activated), the

133 PEVK region attaches itself to the actin filament. Hence, titin's distal Ig region can be

134 pulled not only in the same direction (Fig. 1, middle) as the crossbridges $\left(f_{\text {pass }}>0\right)$, but

135 also in the opposite (Fig. 1, bottom) direction $\left(f_{\text {pass }}<0\right)$. Resultant, $f_{\text {pass }}$ is a function of

$136 l_{h s}$ and $l_{h s[0]}$ as described in the next section.

137 We assumed that skinned myofibril passive tension is induced by titin (Linke et al.,

138 1998). With a typical value of maximum active muscle tension of $20 \mathrm{~N} / \mathrm{cm}^{2}$, we

139 translated measured myofibril tension (Linke et al., 1998) into normalized tension

140 corresponding to normalized titin force. Further length data (Linke et al., 1998) yielded

141 force-elongation data for the entire molecular spring and for the distal Ig and PEVK

142 regions (Fig. 2). We used lookup tables and linearly interpolated desired values from

143 these essentially nonlinear data which were extrapolated linearly when necessary.

145 2.2 The titin-induced 'sticky-spring' mechanism

146 Consider a rectangular adhesive tape stuck on a plain surface. It can bear high forces

147 if pulled along its length axis away from its adherend (stretch), but is easily peeled off

148 in the opposite direction (shortening). We suggest that titin's PEVK region may

149 function similarly during muscular contraction.

150 Along its length, the PEVK region seems to be able to attach to the actin filament, to

151 quickly reattach itself when the bond is broken, and moreover, the attachment may bear

152 an average maximum force $F_{[a m]}$ of $8 \mathrm{pN}$ (Bianco et al., 2007). In the active state, we

153 assume that PEVK-actin attachment occurs spontaneously when the PEVK region 
154 approaches a myosin binding site on actin, and that the attachment can bear $F_{[a m]}$ in any

155 direction (i.e. in our model in any of the two longitudinal half-sarcomere directions).

156 To normalize the force induced by the 'sticky-spring' mechanism, we related $F_{[a m]}$

157 to $F_{[i m]}$. With assumed $20 \mathrm{~N} / \mathrm{cm}^{2}$ maximum isometric active muscle tension and a $42 \mathrm{~nm}$

158 distance between myosin filaments in the crystalline structure of skeletal muscle, the

159 maximum active force related to one myosin is $300 \mathrm{pN}$. Six titin molecules connect

160 each myosin filament to the Z-disc at the corresponding actin filaments. Hence the

161 maximum isometric force related to one titin molecule is $50 \mathrm{pN}$, and $F_{[a m]}=8 \mathrm{pN}$

162 corresponds to $f_{[a m]}=0.16$ normalized maximum attachment force.

163

$164 \quad$ 2.2.1 Single active stretch

165 First, assume a high calcium concentration and that the PEVK region is attached to

166 actin thereby dramatically reducing the free molecular spring length to the distal Ig

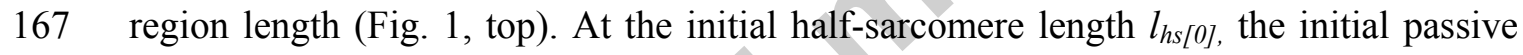

168 half-sarcomere force $f_{\text {pass [0] }}$ acts in the PEVK region as well as in the proximal and

169 distal Ig regions. However, no force pulls on the PEVK-actin attachments. When the

170 active sarcomere is stretched, the force in the distal Ig region increases due to a pull on

171 the most distal (nearest to myosin) PEVK-actin attachment. As soon as the increase in

172 force exceeds the normalized attachment force $f_{[a m]}$, the attachment may break and

173 PEVK may reattach at the same binding site, thus reducing the PEVK chain length

174 between the two most distal PEVK-actin attachments and leading to an additional force

175 bearing of the second (from distal) attachment site. If the stretch continues, more and

176 more of the initial PEVK-actin attachments will bear force, and the elongating PEVK

177 region will approach additional binding sites (Fig. 1, middle). Hence, during the stretch,

178 the PEVK region may be seen as a 'sticky-spring', and its distal-end force $f_{\text {sticky }}$ is the 
179 sum of the constant $f_{\text {pass }[0]}$ (compare appendix B) and the cumulated force pulling on the

180 PEVK-actin attachments $f_{\text {att_cum }}$ (Fig. 1, middle). Hence

181

182

$$
f_{\text {pass }}=f_{\text {sticky }}=f_{\text {pass }[0]}+f_{\text {att_cum }} .
$$

183

184 Note that the sum of these forces does not indicate simultaneous parallel action, but a 185 sequential increase of passive force.

186 We derived a constant stiffness $k_{[s t i c k y]}$ dependent on $l_{h s[0]}$ to characterize the increase

187 in force due to 'sticky-spring' elongation (see appendix B). The half-sarcomere length

188 change equals the sum of the PEVK region's elongation $\Delta l_{P E V K}$ equaling $\Delta l_{\text {sticky }}$ and the

189 distal Ig region's elongation $\Delta l_{\text {dist_lg }}($ Fig. 1, middle):

190

192

193

where $l_{\text {dist } I g}$ is the length of the distal Ig region and $l_{\text {dist } \_g[0]}$ is its length attained under $f_{\text {pass }[0] \text {. }}$

$$
l_{h s}-l_{h s[0]}=\Delta l_{\text {sticky }}+\Delta l_{\text {dist_Ig }}=f_{\text {att_cum }} / k_{[\text {sticky }]}\left(l_{h s[0]}\right)+l_{\text {dist_ } I g}-l_{\text {dist_Ig[0] }},
$$

197 Here, we only consider the case where the initial passive force is lower than $f_{\text {[am] }}$ which, according to the used literature data, corresponds to half-sarcomere lengths below $1.66 \mu \mathrm{m}\left(f_{l}=.31\right.$, descending limb of active force-length relation). Such lengths are exceeded only by a few muscles within their operating ranges (Burkholder and

201 Lieber, 2001). Again, assume that the PEVK region is attached to actin (Fig. 1, top) and further, that the most distal PEVK-actin attachment coincides with the distal end of the 
ranges with 'sticky-spring' effects on active and passive half-sarcomere forces. In the

205 first phase (Fig. 3, top), shortening leads to a stronger decrease in passive force compared to passive shortening since the molecular spring length is reduced to the length of the distal Ig region. Hence

208

209

$$
l_{\text {dist_Ig }_{-} g}=l_{\text {dist_ } I g[0]}-\left(l_{h s[0]}-l_{h s}\right) .
$$

211 The 'sticky-spring' force equaling the passive force is then given by the force-length data of the distal Ig region. Note that the PEVK region is pre-stretched by $f_{\text {pass }[0]}$ before attaching itself to actin. Therefore, as $f_{\text {sticky }}$ decreases to zero during shortening to the

214 first shortening phase's final length $l_{h s[1]}$, the resulting force pulling on the most distal PEVK-actin attachment towards the Z-disc increases from zero to $f_{\text {pass }[0] \text {. }}$

216 During the second shortening phase (Fig. 3, middle), a passive tensile force 217 counteracting the active force develops in the distal Ig region. Notably, it also pulls 218 solely on the most distal PEVK-actin attachment towards the Z-disc. Consequently, the

219 PEVK-actin attachments break consecutively whenever this force exceeds $f_{[\text {break }]}=f_{[a m]}$

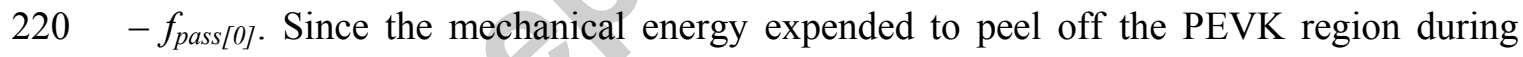
221 half-sarcomere shortening increased approximately with the square of the displacement 222 (Fig. C.1B), we assumed the 'sticky-spring' peel-off force to increase linearly with half223 sarcomere shortening $l_{h s[1]}-l_{h s}$ with a slope of $k_{\left[s h o r t \_2\right]}$ depending on $l_{h s[0]}$ (see appendix 224 C):

$$
f_{\text {pass }}=-f_{\text {sticky }}=-k_{[\text {short_2 }]}\left(l_{h s[0]}\right) \cdot\left(l_{h s[1]}-l_{h s}\right) .
$$

228 The negative sign indicates that the 'sticky-spring' force opposes the active force $f_{\text {act }}$. 
Myosin entering the range of the attached PEVK region at the length $l_{h s[1]}$ reduces the number of effective crossbridges, thereby decreasing active forces compared to the crossbridge and sliding filament theories. The $f_{l}$ value decrease is defined by the region $l_{\text {block }}$ (compare appendix A) where potential crossbridges are inhibited by the attached PEVK region (Fig. 3, middle):

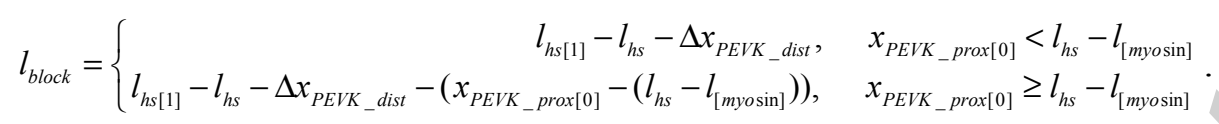

The proximal displacement of the attached PEVK region's distal end $\Delta x_{P E V K}$ dist (Fig. 3, middle) is given by

$$
\Delta x_{P E V K_{-} d i s t}=\left\{\begin{array}{rr}
0, & l_{h s[1]}-l_{h s}<l_{\left[d i s t_{-} I g_{-} b r e a k\right]}, \\
s\left(l_{h s[0]}\right) \cdot\left(l_{h s[1]}-l_{h s}-l_{\left[d i s t_{-} I g_{-} b r e a k\right]}\right), & l_{h s[1]}-l_{h s} \geq l_{\left[d i s s_{-} I g_{-} b r e a k\right]}
\end{array}\right.
$$

where $s\left(l_{h s[0]}\right)$ (see appendix C) is a gearing $<1$ relating the length change of the half-

$\left.244 f_{[b r e a k]}\right)$ to $\Delta x_{P E V K_{-} \text {dist }}$. The constant length $x_{P E V K \text { prox }[0]}$ corresponds to the distance between the Z-disc and the proximal end of the PEVK region, and $l_{\text {[myosin] }}(0.8 \mu \mathrm{m})$ is the length of half a myosin molecule.

The third shortening phase (Fig. 3, bottom) begins when the distal Ig region's distal end passes the PEVK region's proximal end at the corresponding length $l_{h s[2]}$. In this

249 situation the distal Ig region is pre-stretched to $l_{\text {dist } I g[2]}$ with the force $f_{\text {sticky[2] }}\left(<f_{[\text {break }]}\right)$.

250 We assume that during half-sarcomere shortening $l_{h s[2]}-l_{h s}$ the PEVK region attaches as described in section 2.2.1 and that similar equations apply, however, resulting here in passive force counteracting the active force. Remaining initial PEVK-actin attachments 
253 continue to break consecutively during shortening, thus, contrary to stretch, no initial

254 PEVK-actin attachments can be recruited permanently for force bearing of the 'sticky-

255 spring'. Thus, 'sticky-spring' force only increases due to approaching new binding sites

256 on actin at intervals $c_{[b i n d]}(0.038 \mu \mathrm{m})$, and $k_{[s t i c k y]}$ equals $f_{[a m]} / c_{[b i n d]}$ (compare Fig.

257 B.1A). Neglecting the decrease in force distal to the first newly approached binding site

258 due to its minor role in passive force generation, the passive force equals (compare Eq.

$259(3))$

260

261

$$
f_{\text {pass }}=-f_{\text {sticky }}=-\left(f_{\text {sticky[2] }}+f_{\text {att_cum }}\right) .
$$

262

The proximal displacement of the PEVK region's proximal end $\Delta x_{P E V K \_ \text {prox }}$ (Fig. 3,

264 bottom) is defined by $k_{[s t i c k y]}$. Hence (compare Eq. (4)),

$$
l_{h s[2]}-l_{h s}=\Delta x_{P E V K_{-} \text {prox }}+\Delta l_{\text {dist_Ig }}=f_{\text {att_cum }} / k_{[s t i c k y]}+l_{\text {dist_Ig }}-l_{\text {dist_Ig[2] }} .
$$
proximal PEVK end. Hence

$$
l_{\text {block }}=\left\{\begin{aligned}
l_{\text {block }[2]}+.5 \cdot \Delta x_{\text {PEVK_prox }}, & \Delta x_{\text {PEVK_prox }}<2 \cdot l_{\text {block }[2]} \\
\Delta x_{P E V K_{-} \text {prox }}, & \Delta x_{P E V K_{-} \text {prox }} \geq 2 \cdot l_{\text {block }[2]}
\end{aligned}\right.
$$

274 where $l_{\text {block [2] }}$ corresponds to the length $l_{\text {block }}$ at the end of the second shortening phase. 
The 'sticky-spring' mechanism as modeled is independent of stretch or shortening velocity. Consequently, we show forces during active stretch (Fig. 4) and during active shortening (Fig. 5) from different initial lengths in force vs. half-sarcomere length plots. The $f_{v}$ value resulting from the force-velocity relation modulating the active halfsarcomere force component is, however, set to 1 (isometric condition). This approach enables the reading of force enhancement during stretch and force depression during shortening, as well as the reading of residual force enhancement and residual force depression at any half-sarcomere length.

'Sticky-spring' stiffness in stretch experiments increases with increasing initial halfsarcomere length (Fig. B.1A).

\section{Discussion}

In addition to the crossbridge cycle, we proposed a titin-induced molecular 'stickyspring' mechanism to contribute to sarcomere force generation. When compared to passive half-sarcomere length changes, it increases passive force during and after active stretch (Fig. 4, top), and it produces decreased or negative passive force during and after active shortening (Fig. 5, top). Moreover, during shortening, the 'sticky-spring' mechanism interferes with active force production by inhibiting crossbridges (Fig. 5, middle). The mechanism accounts for many experimental observations concerning force-enhancement and force-depression effects leaving the sliding filament and the crossbridge theories intact (i.e. without increasing or decreasing the rate functions of attachment or detachment of myosin heads to actin). Notably, we used only experimental data from the literature to derive 'sticky-spring' forces.

$300 \quad$ Residual force enhancement and residual force depression depend on the muscle's 301 working range. More precisely, no residual force enhancement (Edman et al., 1982;

302 Morgan et al., 2000; Peterson et al., 2004) and no residual force depression (Gordon et 
303 al., 1966; Morgan et al., 2000) were found in the steep range of the ascending limb of

304 the active force-length relationship. Furthermore, residual force enhancement increased

305 from the upper part of the ascending limb to longer lengths (e.g. Herzog et al., 2008;

306 Lee and Herzog, 2008). Predictions of the 'sticky-spring' mechanism reflect these

307 findings. There is no titin length change in the range of the steep part of the ascending

308 limb of the active force-length relationship (myosin hits Z-disc), and thus neither

309 residual force enhancement nor residual force depression are predicted for contractions

310 within this length range. Predicted residual force enhancement increases from the upper

311 part of the ascending limb to longer lengths (Fig. 4) due to increasing 'sticky-spring'

312 stiffness (Fig. B.1A, inset).

313 Measured residual force depression D (Edman, 1975; $20 \%$ for $.15 \mu \mathrm{m}$ half-

314 sarcomere shortening to plateau) is lower by an order of magnitude than residual force

315 enhancement (Herzog et al., 2008; $170 \%$ for $.35 \mu \mathrm{m}$ half-sarcomere stretch from

316 plateau). The 'sticky-spring' mechanism predictions are similar to these experimental

317 results (Figs 4, 5).

318 Sarcomere lengths, though inhomogeneous before stretch, were shown to

319 homogenize during and after stretch on the descending limb of the active force-length

320 relationship (Herzog et al., 2008). Stunningly, this is contrary to what would be

321 expected according to the sliding-filament and the crossbridge theories. The longer the

322 sarcomere, the weaker it should be and should elongate more rapidly until it is 'caught'

323 by passive forces at long lengths which is not seen in the cited experiments. In general,

324 the 'sticky-spring' mechanism leads to higher passive forces during and after active

325 stretch compared to during and after passive stretch. The higher passive stiffness in

326 initially longer sarcomeres, and accordingly the higher passive force during and after

327 stretch in those sarcomeres could result in a compensation of lower active forces 
328 compared to initially shorter sarcomeres and lead to homogenization of sarcomere

329 lengths as observed.

330 For large magnitude stretches, residual force enhancement can reach a plateau

331 (Bullimore et al., 2007) whose development is within the framework of the proposed

332 mechanism. During a stretch, the force gradient in the PEVK region might reach the

333 proximal (to Z-disc) PEVK-region end. Further stretch would then lead to movement of

334 the entire PEVK region along actin at a rather constant force (neglecting the increase in

335 passive force in the proximal Ig region). The lower the bearable PEVK-actin attachment

336 force and the shorter and the stiffer the PEVK region, the lower the stretch magnitude at

337 which the plateau occurs and the lower the plateau force.

338 Moreover, residual force enhancement (Edman et al., 1982) as well as residual force

339 depression (Herzog and Leonard, 1997) do not disappear if force temporarily decreases

340 to zero during stretch or shortening, but vanish if activation temporarily decreases to

341 zero after the final stretch or shortening length is reached. With regards to the 'sticky-

342 spring' mechanism, this also makes perfect sense because it is independent of a decrease

343 in force to zero induced by a quick shortening step. On the other hand, when the

344 sarcomere deactivates, the PEVK-actin attachments must break according to our theory,

345 otherwise the proposed mechanism would be a one-way street. We expect tropomyosin

346 to break the PEVK-actin attachments while covering the myosin binding sites on actin.

348 Model limitations

349 Though many experimental results might be explained qualitatively with the mechanism

350 proposed in its current form, a well-documented feature of force depression is not yet

351 included. Similar to experiments by Marechal and Plaghki (1979), the 'sticky-spring'

352 mechanism leads to residual force depression which linearly depends on the shortening

353 distance in a number of ranges (Fig. 6). However, residual force depression not only 
354 depends on shortening distance, but also on shortening velocity (Marechal and Plaghki, 355 1979).

356 Considering the narrow interfilamentary space, it may be possible that instead of 357 sliding past them, the myosin filaments knock off a fraction of PEVK-actin attachments 358 during shortening. The impulse exerted on the PEVK-actin attachment increases with 359 increasing contraction velocity. Hence, the probability of knock-off might increase with 360 velocity resulting in decreased force depression. To incorporate the speculated knock-

361 off into the model in a simple manner, we could assume that if the most distal PEVK-

362 actin attachment is knocked off at the beginning of the second shortening phase (Fig. 3,

363 middle), all following PEVK-actin attachments of this particular titin molecule will also

364 be knocked-off. Then, a factor decreasing from one towards zero with increasing 365 velocity can simply be multiplied with the passive force in the second and the third 366 shortening phases as well as with the length $l_{\text {block }}$ of PEVK-inhibited binding sites to 367 account for velocity-dependent force depression. Interestingly, the fraction of knocked368 off PEVK-actin attachments might increase with a decreasing $f_{[b r e a k]}$. Thus, the current 369 model yields the maximal force depression to be expected (Fig. 5), which may decrease 370 with increasing velocities as stated in the literature, and moreover with decreasing $f_{\text {[break] }}$ 371 (i.e. with increasing initial length or after an active pre-stretch).

372 During active isovelocity stretches, muscle stiffness was constant and greater than 373 zero, independent of stretch velocity (Edman et al., 1982) and also independent of initial 374 length (Till et al., 2008). The widely accepted product approach for the active force (Eq.

375 (2)) leads to different active force slopes for different constant velocities in ranges of 376 non-zero force-length relationship slopes, whereas the 'sticky-spring' mechanism in its 377 current form yields passive forces which are independent of stretch velocity. Half378 sarcomere force is the sum of active force and 'sticky-spring' force. Thus the 379 experiments in the range of the plateau conducted by Edman et al. (1982) might be 
380 explained, but those by Till et al. (2008) cannot be explained without accounting for a

381 stretch-velocity dependence in the 'sticky-spring' mechanism model.

382 The increase in passive force attributed to increased titin stiffness in the presence of 383 calcium was shown to be very small (e.g. Labeit et al., 2003; Joumaa et al., 2008) and 384 would not be able to predict residual force enhancement values observed in 385 experiments. However, Labeit et al. (2003) removed actin, and Joumaa et al. (2008)

386 removed the tropomyosin motor troponin C. As a result, no myosin binding-sites on 387 actin were available for the suggested PEVK-actin attachments which, in turn, are 388 crucial for the 'sticky-spring' mechanism. However, the 'sticky-spring' forces depend 389 on the force-elongation relations of distinct titin regions. Stiffness adaptations of these 390 regions due to calcium increase may be worth considering, but were not accounted for 391 in the model due to lack of data.

Functional implications

394 The proposed mechanism strongly links force enhancement with force depression effects, thereby implying functional consequences.

396 Passive, elastic force enhancement might improve the muscle's efficiency in cyclic

397 stretch-shortening situations by temporarily storing and subsequently releasing elastic 398 strain energy (Lindstedt et al., 2002; Pinniger et al., 2006). In a study with rats 399 conducted by Lindstedt et al. (2002), eccentric muscle training increased muscle 400 stiffness, but did not increase the rats' maximum isometric force, thus the ability of 401 muscles to produce force enhancement (perhaps accompanied by force depression) 402 seemed to be a plastic feature of muscles depending on their use. In this context, force 403 depression could be seen as an unwanted by-product of force enhancement, and might 404 be circumvented by timely deactivation in the shortening phase. 
If the muscle's primary function is to produce positive work (i.e. active fiber shortening is the predominant action), the 'sticky-spring' mechanism is contra-indicated since by decreasing the muscle's force it leads to a reduction of the positive work. Muscle fibers and entire muscles showing no force enhancement exist (Pinniger et al., 2006; Stienen et al., 1992) and, following the proposed mechanism, they might lack force depression and might be adapted for production of positive work. mechanism's speculated adaptiveness might be the structural difference of heart titin when compared to skeletal muscle titin. The heart is one of the above mentioned muscles whose primary function is to produce positive work. The PEVK region in heart titin compared to that in selected skeletal muscles is very short (Linke et al., 1996), which is appropriate since it reduces the number of possible PEVK-actin connections and, therefore, reduces force depression.

Active shortening-stretch and stretch-shortening experiments

Non-commutativity of active shortening-stretch vs. stretch-shortening experiments experiments the initial conditions for the second part of the experiment differ from single stretch or single shortening.

In an equidistant shortening-stretch experiment, shortening to an extent where the distal end of the attached PEVK region remains in place leads to equal configuration of 427 the 'sticky-spring' at the final length compared to a purely isometric contraction at the 428 same final length. Therefore, the steady-state force for both experiments may remain 429 unchanged as reported (Herzog and Leonard, 2000). Greater shortening-stretch 
430 amplitude can lead to the development of a force gradient in the PEVK region during

431 stretch and, therefore, to force enhancement.

432 However, the situation for an equidistant stretch-shortening experiment of

433 comparable amplitude would be different from the one already discussed. Due to

434 stretch, the bound PEVK region elongates distally. During the first shortening phase,

435 while the passive force decreases to zero, the force gradient in the PEVK region

436 reverses with only marginal proximal displacement of the PEVK-region's distal end.

437 Thus, the distal PEVK-region end is more distal when the final length is reached than it

438 is in a purely isometric contraction at the same final length. As a result, the steady-state

439 force at the final length is lower than the force resulting from the purely isometric

440 contraction due to decreased passive force or even negative passive force and inhibited

441 crossbridges (i.e. the 'sticky spring' introduces hysteresis).

The force decay after active stretch seen in experimental data

444 The force increase during active myofibril or muscle stretch can be separated into

two main parts with different slopes. Pinniger et al. (2006) related the first part to

increasing force-velocity values, and mainly associated the second part with increased

titin stiffness. The force decay in a subsequent active isometric phase exceeds the force increase in the first part of the active stretch (e.g. Pinniger et al., 2006). This suggests that the force output of the 'sticky-spring' mechanism should decay in isometric conditions. It should be noted that this decay is incompatible with the 'sticky-spring' behavior as modeled.

Interestingly, it has been reported that, depending on force, the Ig regions unfold and refold in a statistical process (e.g. Linke and Grutzner, 2008). Assuming that the 'stickyspring' mechanism is valid, force levels in the distal Ig region can vastly exceed those

455 introduced by passive extension (Fig. 4) during and after active stretch. Therefore, the 
unfolding of the distal Ig region might play a crucial role in the force decay after active stretch. Accounting for such a process, the forces produced by the 'sticky-spring' mechanism would inherit a velocity-dependence and force would decay in the isometric phase after stretch.

Finally, in our model we did not account for increased passive titin stiffness due to high calcium concentrations (Labeit et al., 2003) since to date it is not clear to what extent which titin region stiffens. However, stiffening of mainly the distal Ig or the PEVK regions, for example, might have a profound effect on 'sticky-spring' force output during active stretch which, in turn, might lead to changed folding and unfolding ratios and thereby influence overall time constant(s) of force decay.

\section{Acknowledgement}

We thank the German Science Foundation (DFG) for support of work (SI841/2-2).

\section{APPENDIX A Calculation of $f_{l}$}

We used the rat's actin length (1.04 $\mu \mathrm{m}$; Walker and Schrodt, 1974), the typical myosin length $(1.6 \mu \mathrm{m})$, the measured plateau width $(0.25 \mu \mathrm{m})$, and the measured length of zero active force $(1.27 \mu \mathrm{m})$ of the active sarcomere force-length relationship (Gordon et al., 1966) to calculate the potential range of crossbridges $l_{\text {bind pot }}$ for a given half-sarcomere length. The effective myosin bare-region length was assumed to correspond to half the plateau width, thus the maximal range of operative crossbridges $477 l_{[\text {bind_max }]}$ per half-sarcomere is $1.6 / 2-.25 / 4=.7375[\mu \mathrm{m}]$. In the range of the upper part 478 of the ascending limb, actin filaments entering the range of myosin heads of the 479 adjacent half-sarcomere are supposed to reduce the number of potential crossbridges by 480 half, thus the fraction of $l_{\text {bind pot }}$ in this range is halved. Subtraction of the length $l_{\text {block }}$ of 
481 PEVK-inhibited binding sites (compare Fig. 3) from $l_{\text {bind_pot }}$, and division by $l_{[\text {bind_max] }}$

482 yielded the corresponding $f_{l}$ value.

483

484 APPENDIX B Derivation of 'sticky-spring' stiffness during stretch

485 Consider a long active stretch of the activated half-sarcomere. The attached PEVK 486 region elongates along actin and the force pulling on the distal PEVK region end 487 increases due to 1) consecutive force bearing from distal to proximal PEVK segments in 488 between initial PEVK-actin attachments and 2) superimposed force steps induced by 489 binding sites approached during elongation.

490 Using the given passive force at any initial half-sarcomere length, we determined the 491 initial PEVK-region length, $l_{P E V K[0]}$. Dividing $l_{P E V K[0]}$ by $c_{[b i n d]}$, the distance between 492 two binding sites on actin $(0.038 \mu \mathrm{m})$, and rounding up to the next integer yielded the 493 number of initial PEVK-actin attachments. For simplicity, we assumed that the 494 proximal end of the PEVK region would coincide with a PEVK-actin attachment. This 495 led to full loading of all initial PEVK-actin attachments from the distal to the proximal 496 end only when 'sticky-spring' force exceeded $2.5 F_{[i m]}$. Hence, the proximal Ig region 497 did not elongate during stretch.

498 To calculate the 'sticky-spring' force-elongation relation (Fig. B.1A), we elongated 499 the distal PEVK end by defined steps $\Delta x_{i}$ and iterated the corresponding increased distal 500 force $f_{i}$ (Fig. B.1B). The length change $\Delta l_{k}$ of the $k$-th recruited PEVK segment (i.e. 501 PEVK segment loaded during active stretch) due to the segment-force increase from the

502 force prior to the step $f_{i-1}-k f_{[a m]}$ to the force after the step $f_{i}-k f_{[a m]}$ is 503

$$
\Delta l_{k}=c_{[b i n d]} \cdot\left(\frac{l_{P E V K}\left(f_{i}-k \cdot f_{[a m]}\right)}{l_{P E V K}\left(f_{i-1}-k \cdot f_{[a m]}\right)}-1\right) .
$$


The expression in large parentheses yields the strain of the respective segment,

507 which was calculated using the passive PEVK force-length relation (Fig. 2). Throughout

508 appendices $\mathrm{B}$ and $\mathrm{C}$, lengths depend on the forces written in parentheses. Each $\Delta l_{k}$

509 migrates to the distal end and is finally strained by the force $f_{i}$. It is multiplied with a

510 factor calculated again using the PEVK force-length relation to yield its final length. $\Delta x_{i}$

511 is then expressed by

512

$$
\Delta x_{i}=l_{\text {end }}\left(f_{i-1}\right) \cdot\left(\frac{l_{P E V K}\left(f_{i}\right)}{l_{P E V K}\left(f_{i-1}\right)}-1\right)+\sum_{k=1}^{\text {recr-segs }}\left(\Delta l_{k} \cdot \frac{l_{P E V K}\left(f_{i}\right)}{l_{P E V K}\left(f_{i}-k \cdot f_{[a m]}\right)}\right),
$$
most distal PEVK-actin attachment and the PEVK-region's distal end with length $l_{\text {end }}<$

$c_{[b i n d]}$. The recr_segs is the number of segments recruited for force-bearing by the ‘sticky-spring' mechanism (Fig. B.1B).

519 Considering the thousands of titin molecules arranged in parallel in a sarcomere and the probability distribution of PEVK-actin attachment force, we approximated the 'sticky-spring' force increase to depend linearly on elongation (Fig. B.1A), resulting in a constant stiffness $k_{[s t i c k y]}$ for each initial half-sarcomere length (Fig. B.1A, inset).

APPENDIX C The second active shortening phase

We calculated discontinuous force-shortening relations during the second shortening phase resulting from 'sticky-spring' peel-off for different initial sarcomere lengths (Fig. C.1A). For this purpose, we used the passive force-length relations of titin, the distal Ig region, and the PEVK region (Fig. 2). The free spring consists of the distal Ig region and a fraction of the PEVK region in series. Its length, $l_{\text {spring }}$, is expressed by 


$$
l_{\text {spring }}=l_{\text {dist_Ig }}\left(f_{\text {sticky }}\right)+n \cdot c_{[\text {bind }]} \cdot \frac{l_{P E V K}\left(f_{\text {sticky }}\right)}{l_{P E V K}\left(f_{\text {pass }[0]}\right)},
$$

where $n$ is the number of PEVK-actin attachments already broken. Force peaks

534 occur due to the breaking of PEVK-actin attachments at $f_{\text {[break] }}$. The breaking of one distal PEVK-actin attachment induces a stepwise proximal displacement of the PEVK-

$$
\Delta l_{[\text {peak }]}=c_{[\text {bind }]} \cdot\left(\frac{l_{P E V K}\left(f_{[\text {break }]}\right)}{l_{P E V K}\left(f_{\text {pass }[0]}\right)}+1\right) .
$$

541 Expected homogenization effects induced by the parallel arrangement of thousands of titin filaments with, for example, distributed PEVK-actin attachment strengths (Bianco et al, 2007), led us to translate the half-sarcomere length change to $\Delta x_{P E V K_{-} \text {dist }}$ via the

544 gearing ratio $s\left(l_{h s[0]}\right)$ :

$$
s\left(l_{h s[0]}\right)=\frac{c_{[\text {bind }]}}{\Delta l_{[\text {peak }]}}=\frac{l_{P E V K}\left(f_{\text {pass }[0]}\right)}{l_{P E V K}\left(f_{\text {pass }[0]}\right)+l_{P E V K}\left(f_{[\text {break }]}\right)} .
$$
approximately quadratic function (Fig. C.1B). To derive $l_{h s[0]}$-dependent $k_{[\text {short_2] }}$, the constant stiffness related to second-phase half-sarcomere shortening $l_{h s[1]}-l_{h s}$ (Fig. 
553 List of symbols and abbreviations

$554 c_{[b i n d]} \quad$ distance of binding sites on actin $(=.038 \mu \mathrm{m})$

$555 f_{\text {act }} \quad$ normalized active half-sarcomere force component

$556 F_{[a m]} \quad$ maximum bearable PEVK-actin attachment force $(=8 \mathrm{pN})$

$557 f_{[a m]} \quad$ normalized $F_{[a m]}(=.16)$

$558 f_{[b r e a k]} \quad$ force in distal Ig region at which PEVK-actin attachment breaks $\left(=f_{[a m]}-\right.$

$\left.559 \quad f_{\text {pass }[0]}\right)$

$560 \quad F_{h s} \quad$ half-sarcomere force

$561 \quad f_{h s} \quad$ normalized half-sarcomere force

$562 F_{[i m]} \quad$ maximum isometric force

$563 f_{l} \quad$ factor due to active force-length relationship

$564 f_{\text {pass }} \quad$ passive half-sarcomere force

$565 f_{\text {pass }[0] \quad \text { initial passive half-sarcomere force }}$

$566 f_{\text {sticky }} \quad$ 'sticky-spring' force (sum of forces pulling on the PEVK region)

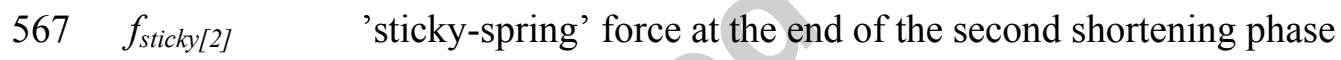

$568 f_{v} \quad$ factor due to active force-velocity relationship

$569 \quad k_{[\text {short_2] }} \quad$ linear stiffness during second shortening phase

$570 \quad k_{[s t i c k y]} \quad$ 'sticky-spring' stiffness during stretch and during third phase shortening

$571 \quad l_{\text {block }} \quad$ length range with PEVK inhibited potential crossbridges

$572 l_{\text {block }[2]}$ length range with PEVK inhibited potential crossbridges at the end of the

573 second shortening phase

$574 \quad l_{\text {bind_pot }} \quad$ potential range of operative crossbridges

$575 l_{[\text {bind_max }] \quad \text { maximal range of operative crossbridges }}$

$576 \quad l_{\text {dist } \_g} I$ length of titin's distal Ig region

$577 l_{\text {dist_Ig[0] }} \quad$ length of titin's distal Ig region attained under $f_{\text {pass }[0]}$

$578 l_{\text {dist_ } I g[2]} \quad$ length of titin's distal Ig region attained under $f_{\text {sticky [2] }}$ 
$579 l_{\text {[dist_Ig_break] }} \quad$ length of titin's distal Ig region attained under $f_{[\text {break] }}$

$580 l_{\text {end }} \quad$ length of PEVK region end in between most distal PEVK-actin

$581 \quad$ attachment and distal PEVK region end

$582 \quad l_{h s} \quad$ half-sarcomere length

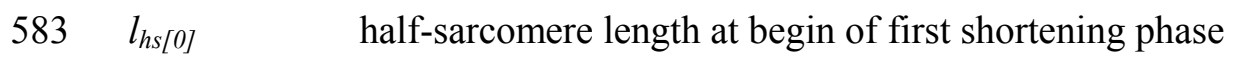

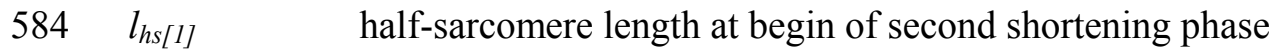

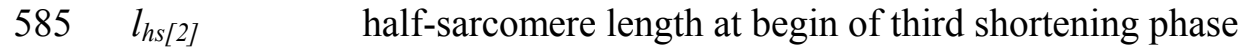

$586 \quad l_{[m y o s i n]} \quad$ half myosin length $(0.8 \mu \mathrm{m})$

$587 \quad l_{P E V K} \quad$ length of titin's PEVK region

$588 l_{P E V K[0]} \quad$ length of titin's PEVK region attained under $f_{\text {pass }[0]}$

$589 \quad n \quad$ number of initially bound PEVK segments

590 recr_segs number of PEVK segments recruited for 'sticky-spring' force bearing

$591 s \quad$ slope of proximal PEVK end length change in second shortening phase

$592 \quad v_{h s} \quad$ half-sarcomere velocity

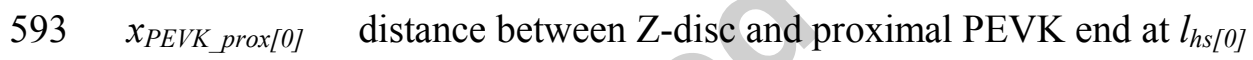

$594 \Delta l_{\text {dist } \_g} \quad$ length change of the distal Ig region

$595 \Delta l_{k} \quad k$-th recruited (loaded during active stretch) PEVK segment in between

596 PEVK-actin attachments (Fig. B.1B, bottom)

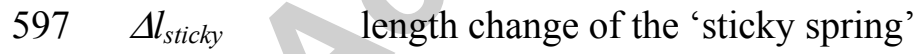

$598 \Delta x_{i} \quad$ defined elongation steps for determination of 'sticky spring' force-

$599 \quad$ elongation relation

$600 \Delta x_{P E V K_{-}}$dist $\quad$ length change of the distal PEVK end during shortening

$601 \Delta x_{P E V K}$ prox $\quad$ length change of the proximal PEVK end during shortening

602 


\section{References}

Abbot, B.C., Aubert, X.M., 1952. The force exerted by active striated muscle during and after change of length. J. Physiol. 117, 77-86.

Bianco, P., Nagy, A., Kengyel, A., Szatmari, D., Martonfalvi, Z., Huber, T., Kellermayer, M.S., 2007. Interaction forces between F-actin and titin PEVK domain measured with optical tweezers. Biophys. J. 93, 2102-2109.

Bullimore, S.R., Leonard, T.R., Rassier, D.E., and Herzog, W., 2007. History-dependence of isometric muscle force: effect of prior stretch or shortening amplitude. J. Biomech. 40, 1518-1524.

Burkholder, T.J., Lieber, R.L., 2001. Sarcomere length operating range of vertebrate muscles during movement. J. Exp. Biol. 204, 1529-1536.

Edman, K.A., 1975. Mechanical deactivation induced by active shortening in isolated muscle fibres of the frog. J. Physiol. 246, 255-275.

Edman, K.A., 1996. Fatigue vs. shortening-induced deactivation in striated muscle. Acta Physiol. Scand. $156,183-192$.

Edman, K.A., Caputo, C., Lou, F., 1993. Depression of tetanic force induced by loaded shortening of frog muscle fibres. J. Physiol. 466, 535-552.

Edman, K.A., Elzinga, G., Noble, M.I., 1982. Residual force enhancement after stretch of contracting frog single muscle fibers. J. Gen. Physiol. 80, 769-784.

Forcinito, M., Epstein, M., Herzog, W., 1998. Can a rheological muscle model predict force depression/enhancement? J. Biomech. 31, 1093-1099., doi:10.1016/S0021-9290(98)00132-8.

Gordon, A.M., Huxley, A.F., Julian, F.J., 1966. The variation in isometric tension with sarcomere length in vertebrate muscle fibres. J. Physiol. 184, 170-192.

Herzog, W., Leonard, T.R., 1997. Depression of cat soleus-forces following isokinetic shortening. J. Biomech. 30, 865-872., doi:10.1016/S0021-9290(97)00046-8.

Herzog, W., Leonard, T.R., 2000. The history dependence of force production in mammalian skeletal muscle following stretch-shortening and shortening-stretch cycles. J. Biomech. 33, 531-542., doi:10.1016/S0021-9290(99)00221-3.

Herzog, W., Lee, E.J., Rassier, D.E., 2006. Residual force enhancement in skeletal muscle. J. Physiol. $574,635-642$.

Herzog, W., Leonard, T.R., Joumaa, V., Mehta, A., 2008. Mysteries of muscle contraction. J. Appl. Biomech. 24, 1-13.

Hill, A.V., 1938. The heat of shortening and the dynamic constants of muscle. Proc. Roy. Soc. Lond. 126, 136-195.

Huxley, A.F., Niedergerke, R., 1954. Structural changes in muscle during contraction; interference microscopy of living muscle fibres. Nature 173, 971-973.

Huxley, H., Hanson, J., 1954. Changes in the cross-striations of muscle during contraction and stretch and their structural interpretation. Nature 173, 973-976.

Huxley, A.F., 1957. Muscle structure and theories of contraction. Progr. Biophys. Chem. 7, 255-318.

Joumaa, V., Rassier, D.E., Leonard, T.R., and Herzog, W., 2008. The origin of passive force enhancement in skeletal muscle. Am J Physiol Cell Physiol 294, C74-8.

Kellermayer, M.S., Granzier, H.L., 1996. Calcium-dependent inhibition of in vitro thin-filament motility by native titin. FEBS Lett. 380, 281-286., doi:10.1016/0014-5793(96)00055-5. 
663

664

665

666

667

668

669

670

671

672

673

674

675

676

677

678

679

680

681

682

683

684

685

686

687

688

689

690

691

692

693

694

695

696

697

698

699

700

701

702

703

704

705

706

707

708

709

710

711

712

713

714

715

716

717

718

719

720

721

722

723

Labeit, D., Watanabe, K., Witt, C., Fujita, H., Wu. Y., Lahmers, S., Funck, T., Labeit, S., Granzier, H., 2003. Calcium-dependent molecular spring elements in the giant protein titin. Proc. Natl. Acad. Sci. USA $100,13716-13721$.

Lee, E.J., Herzog, W., 2008. Residual force enhancement exceeds the isometric force at optimal sarcomere length for optimized stretch conditions. J. Appl. Physiol. 105, 457-462.

Lindstedt, S.L., Reich, T.E., Keim, P., LaStayo, P.C., 2002. Do muscles function as adaptable locomotor springs? J. Exp. Biol. 205, 2211-2216.

Linke, W.A., Ivemeyer, M., Olivieri, N., Kolmerer, B., Ruegg, J.C., Labeit, S., 1996. Towards a molecular understanding of the elasticity of titin. J. Mol. Biol. 261, 62-71., doi:10.1006/jmbi.1996.0441.

Linke, W.A., Grutzner A., 2008. Pulling single molecules of titin by AFM - recent advances and physiological implications. Pflugers Arch. 456, 101-115.

Linke, W.A., Ivemeyer, M., Mundel, P., Stockmeier, M.R., Kolmerer, B., 1998. Nature of PEVK-titin elasticity in skeletal muscle. Proc. Natl. Acad. Sci. USA 95, 8052-8057.

Marechal, G., Plaghki, L., 1979. The deficit of the isometric tetanic tension redeveloped after a release of frog muscle at a constant velocity. J. Gen. Physiol. 73, 453-467.

Meijer, K., Grootenboer, H.J., Koopman, H.F., van der Linden, B.J., Huijing, P.A., 1998. A Hill type model of rat medial gastrocnemius muscle that accounts for shortening history effects. J. Biomech. 31, 555-563., doi:10.1016/S0021-9290(98)00048-7.

Morgan, D.L, Mochon, S., Julian, F.J., 1982. A quantitative model of intersarcomere dynamics during fixed-end contractions of single frog muscle fibers. Biophys. J. 39, 189-196.

Morgan, D.L., 1990. New insights into the behavior of muscle during active lengthening. Biophys. J. 57, 209-221.

Morgan, D.L., Whitehead, N.P., Wise, A.K., Gregory, J.E., Proske, U., 2000. Tension changes in the cat soleus muscle following slow stretch or shortening of the contracting muscle. J. Physiol. 522, 503-513.

Niederlander, N., Raynaud, F., Astier, C., Chaussepied, P., 2004. Regulation of the actin-myosin interaction by titin. Eur. J. Biochem. 271, 4572-4581.

Peterson, D.R., Rassier, D.E., Herzog, W., 2004. Force enhancement in single skeletal muscle fibres on the ascending limb of the force-length relationship. J. Exp. Biol. 207, 2787-2791.

Pinniger, G.J., Ranatunga, K.W., Offer, G.W., 2006. Crossbridge and non-crossbridge contributions to tension in lengthening rat muscle: force-induced reversal of the power stroke. J. Physiol. 573, 627-643.

Prado, L.G., Makarenko, I., Andresen, C., Kruger, M., Opitz, C.A., Linke, W.A., 2005. Isoform diversity of giant proteins in relation to passive and active contractile properties of rabbit skeletal muscles. J. Gen. Physiol. 126, 461-480.

Stienen, G.J., Versteeg, P.G., Papp, Z., Elzinga, G., 1992. Mechanical properties of skinned rabbit psoas and soleus muscle fibres during lengthening: effects of phosphate and Ca2+. J. Physiol. 451, 503-523.

Sugi, H., Tsuchiya, T., 1988. Stiffness changes during enhancement and deficit of isometric force by slow length changes in frog skeletal muscle fibres. J. Physiol. 407, 215-229.

Till, O., Siebert, T., Rode, C., and Blickhan, R., 2008. Characterization of isovelocity extension of activated muscle: a Hill-type model for eccentric contractions and a method for parameter determination. J. Theor. Biol. 255, 176-187.

Walker, S.M., Schrodt, G.R., 1974. I segment lengths and thin filament periods in skeletal muscle fibers of the Rhesus monkey and the human. Anat. Rec. 178, 63-81. 
FIGURE LEGENDS

725 Fig. 1. Schematic of the active (high calcium concentration) half-sarcomere: (top) at an initial length $l_{h s[0]}$ 726 on the descending limb of the active force-length relationship; (middle) after stretch from $l_{h s[0]}$; (bottom)

727 after shortening from $l_{h s[0]}$. All actin, myosin and titin (its molecular spring part is colored) filaments are

728 represented with one specimen each. The long axis length ratios, as well as the number of myosin heads

729 correspond to physiological values. (top) The PEVK region (yellow) is attached to actin at myosin

730 binding sites (black dots). The length of titin's proximal Ig region, PEVK region, and distal Ig region

731 attained under the initial passive force $f_{\text {pass }[0]}$ is depicted. (middle) The sum of the length changes $\Delta l_{P E V K}$

732 and $\Delta l_{\text {dist } I g}$ equals the stretch distance. (middle, enlarged view) The 'sticky-spring' force $f_{\text {sticky }}$ equals the

733 sum of attachment forces $f_{\text {att_i } i}$ pulling on the PEVK-actin attachments and $f_{\text {pass }[0] \text {. (bottom) Inhibition of }}$

734 crossbridges by PEVK-actin attachments is illustrated.

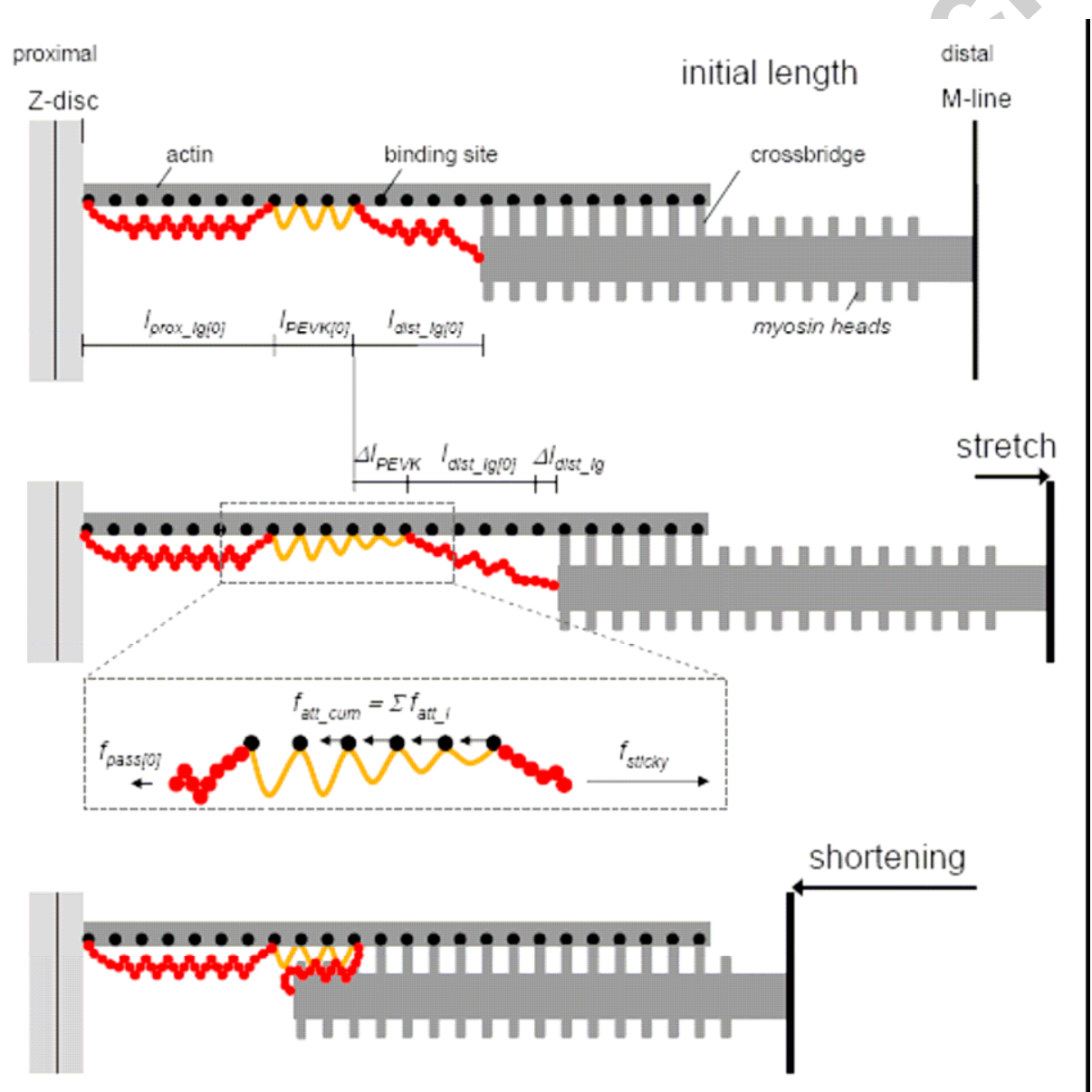


736 Fig. 2. Force-length relations (data adapted from Linke et al., 1998) of titin's entire molecular spring 737 region (x-mark), its PEVK region (square), and its distal Ig region (circle).

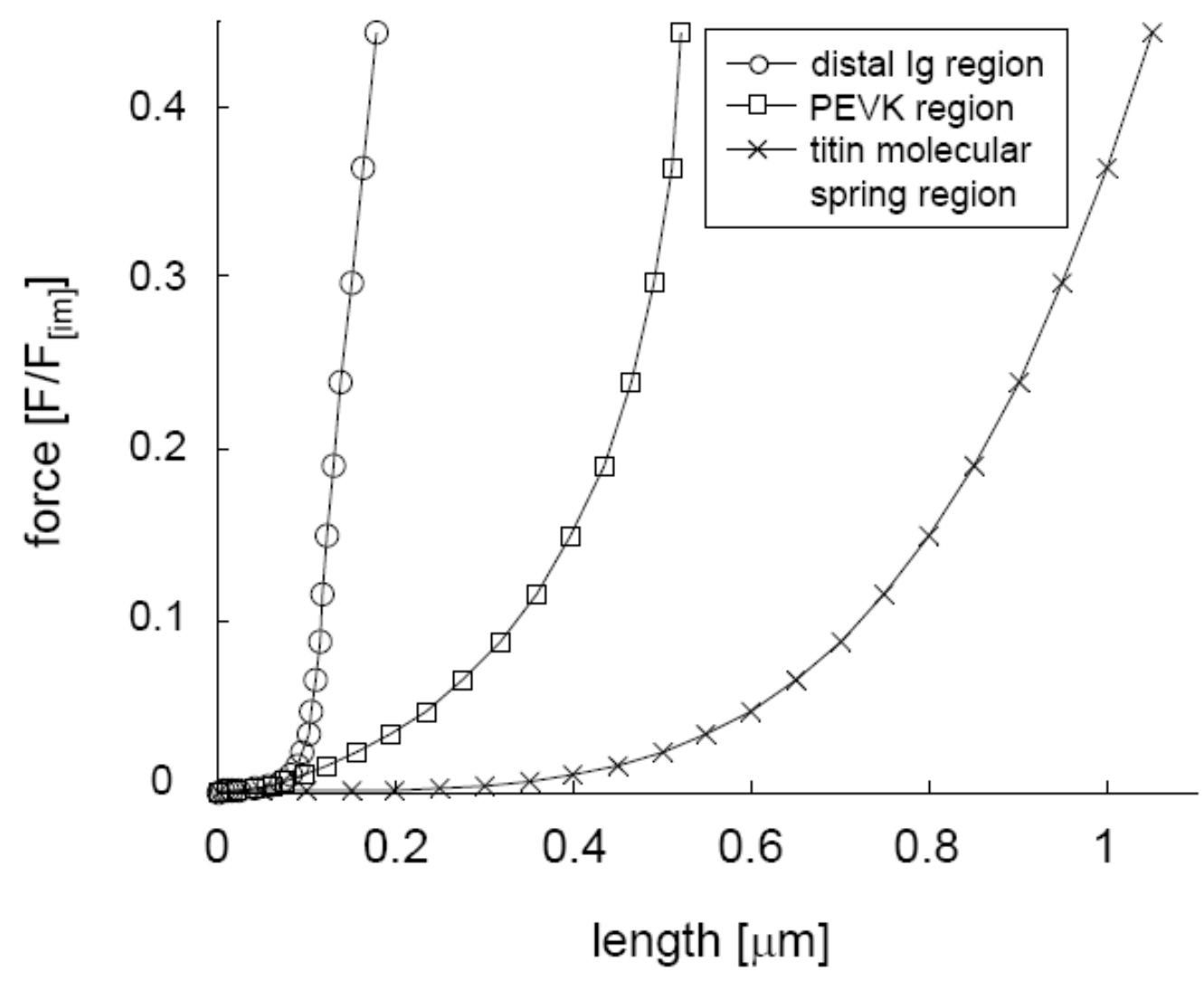

738

Figure 2

739 
739 Fig. 3. Schematic of three subsequent phases (from top to bottom) of active half-sarcomere shortening.

740 Only the vicinity of the myosin end and a part of the actin filament is depicted. The dark grey actin

741 (dotted with binding sites) and myosin segments depict initial loci of the respective phase, and the

742 transition between titin's PEVK region (yellow) and the distal Ig region (red) is marked with a circle

743 containing a dot. The myosin segments depicted in light grey correspond to their position in a later stage,

744 while the PEVK region and distal Ig region are now shown in grey. The arrows indicate length changes.

745 (top) Shortening from the initial length results in quick unloading of the distal Ig region and proximal

746 loading of the most distal PEVK actin attachment. (middle) The myosin end passes the distal end of the

747 attached PEVK region and the attached PEVK region begins to peel off. Furthermore, dependent on the

748 attached PEVK-region length and on the overlap with the myosin end, crossbridges are inhibited in the

749 range $l_{\text {block }}$. (bottom) The sufficiently lengthened, peeled PEVK segment is allowed to reattach behind the

750 attachment of its former proximal end. For further explanations see text. 
active shortening

proximal

distal
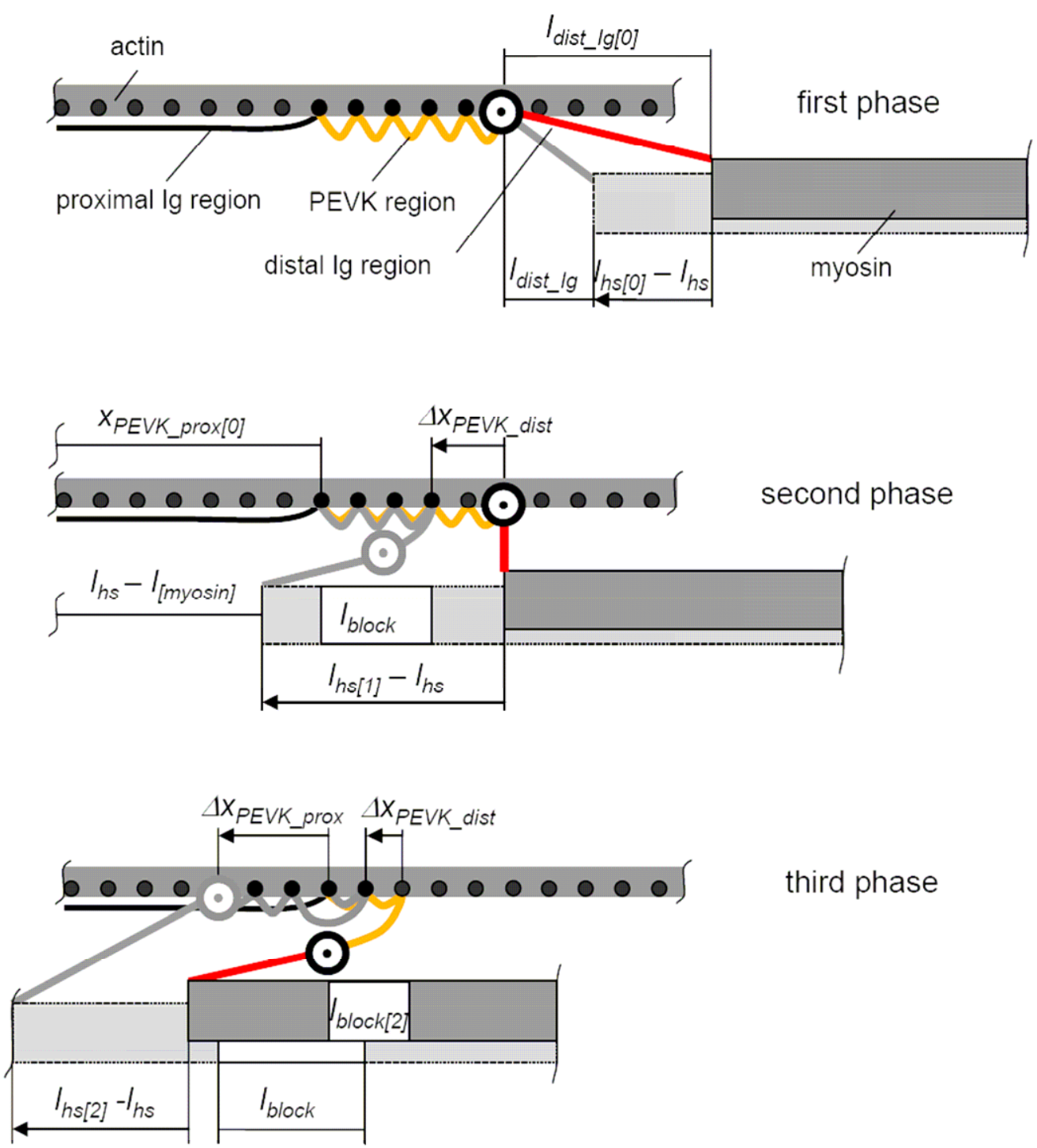

third phase 
752 Fig. 4. Forces during quasi-isometric active stretch (thick lines). From top to bottom, the passive force,

753 the active force, and the sum of the two, the half-sarcomere force, is shown. For orientation, noted with

754 thin black lines, the passive (low calcium concentration) half-sarcomere force (top), the isometric active

755 half-sarcomere force (middle) and both as well as the sum of the two (bottom) are given. 

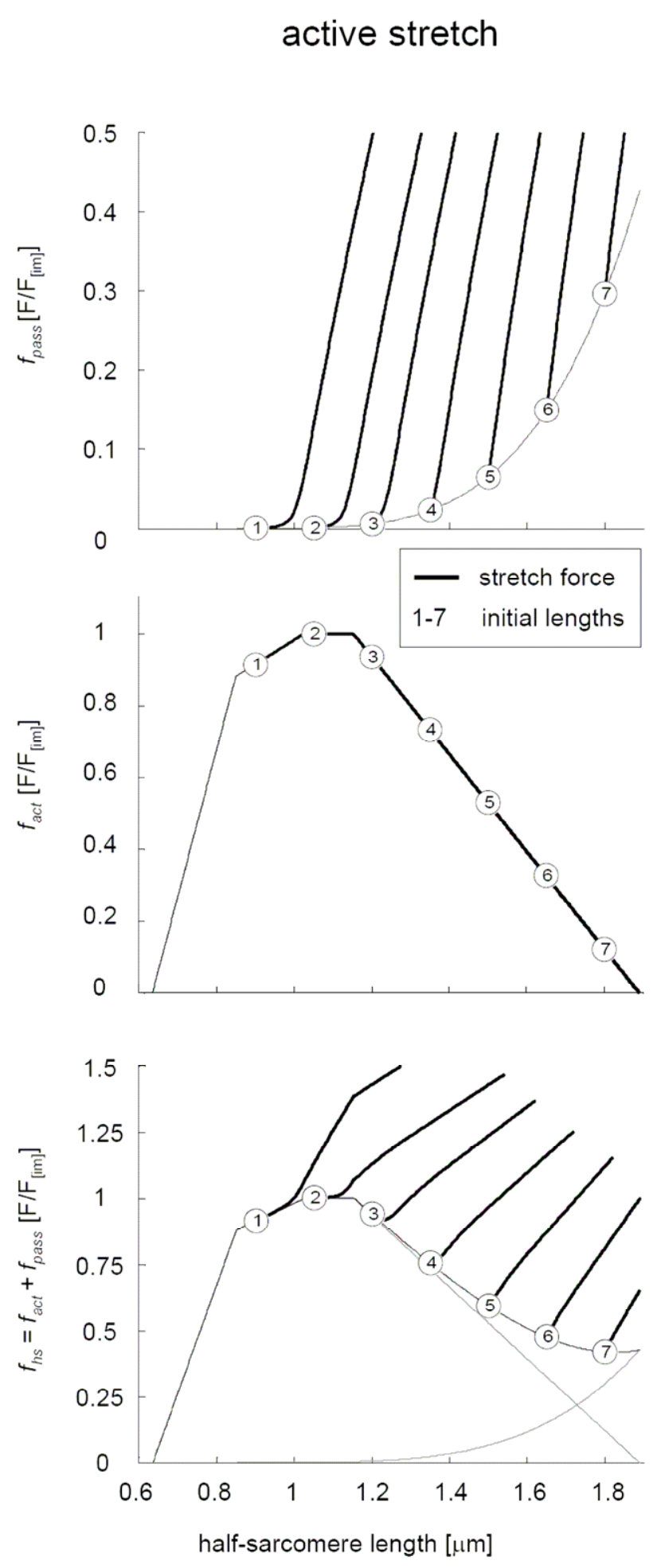

Figure 4 
757 Fig. 5. Forces during quasi-isometric active shortening (thick lines). From top to bottom, the passive

758 force, the active force and the sum of the two, the half-sarcomere force, is shown. The broken black line,

759 the thick black line, and the thick grey line indicate the first, the second, and the third shortening phases,

760 respectively. For orientation, noted with thin black lines, the passive (low calcium concentration) half-

761 sarcomere force (top), the isometric active half-sarcomere force (middle), and both, as well as the sum of

762 the two (bottom) are given. At the length $.85 \mu \mathrm{m}$, indicated by the vertical line, myosin hits the Z-disc. 


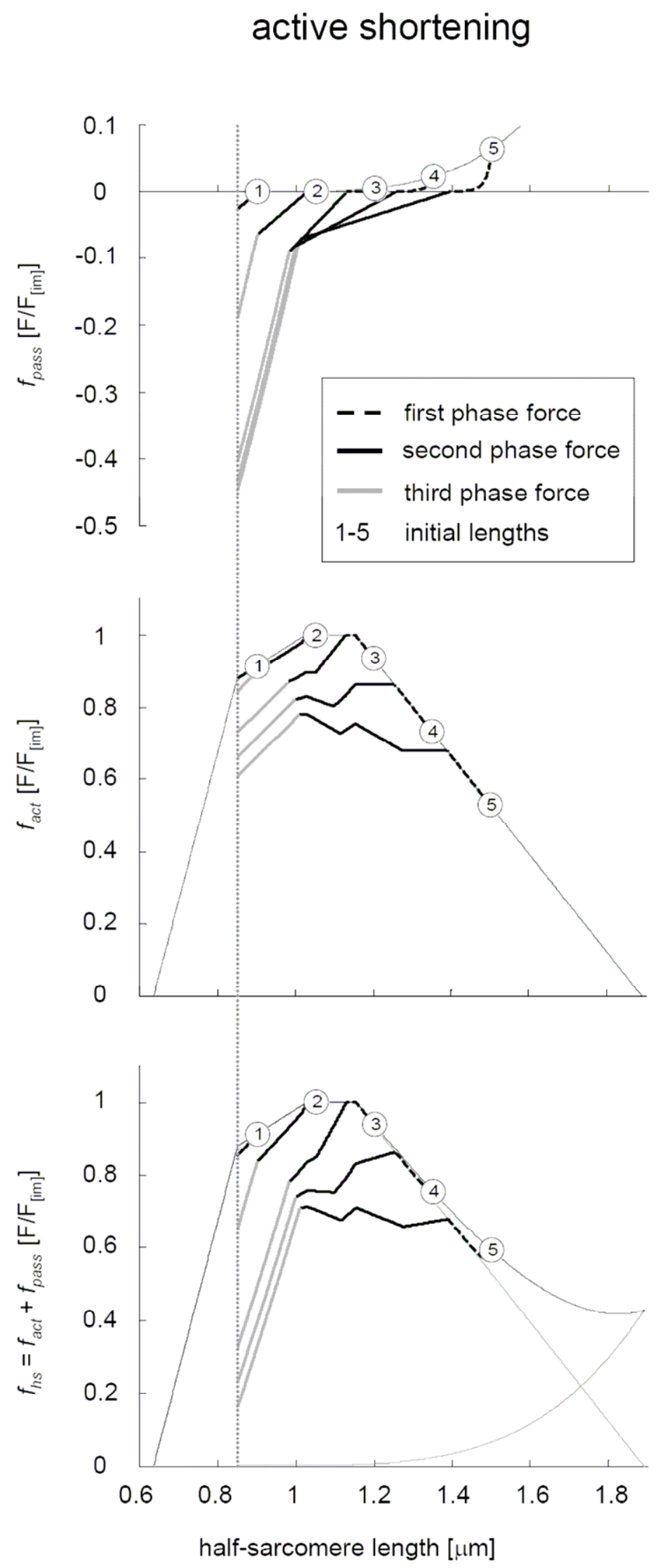

Figure 5 
764 Fig. 6. Residual force depression (RFD). Thick lines show half-sarcomere forces due to quasi-isometric

765 shortening from different (1-9) initial half-sarcomere lengths $l_{h s[0]}$. The vertical, broken line indicates a 766 chosen, final half-sarcomere length. RFD (inset) depends almost linearly on the shortening distance 767 (initial length - final length).

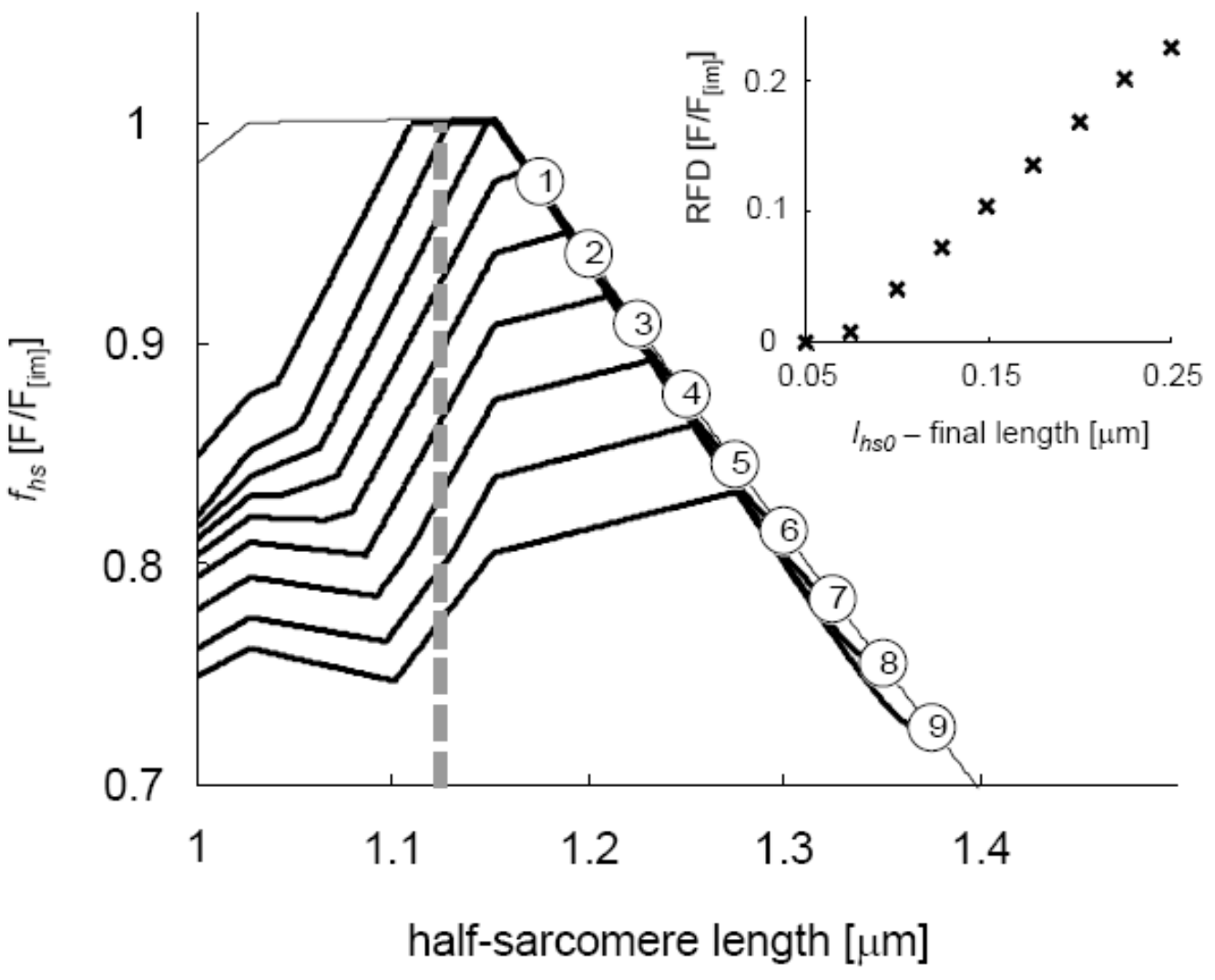


769 Fig. B.1. Linear approximation of 'sticky-spring' stiffness during stretch from different initial half770 sarcomere lengths $l_{h s[0]}$. (A) Discontinuous force-elongation relations of the 'sticky-spring' resulting from 771 stretch (black lines) are approximated linearly (grey lines). (A, inset) The linear 'sticky-spring' stiffness $772 k_{[s t i c k y]}$ increases with increasing $l_{h s[0]}$. Circles correspond to the grey lines in A. (B) Schematic of 'sticky773 spring' elongation by $\Delta x_{i}$. (B, top): The PEVK region is attached to actin. The distal Ig region and the 774 PEVK segments 1 and 2 (encircled numbers) bear forces decreasing successively by the PEVK-actin 775 attachment force $f_{[a m]}$. The grey springs only bear the initial passive force $f_{\text {pass[0] }}$. (B, bottom) Stretch by $776 \Delta x_{i}$ results in the same force increase in the distal PEVK-region end $l_{\text {end }}$ and in segments 1 and 2 . In this 777 example, during stretch, the force difference between segments 2 and 3 exceeded $f_{[a m]}$, and segment 3 778 now bears force exceeding $f_{\text {pass }[0]}$. 


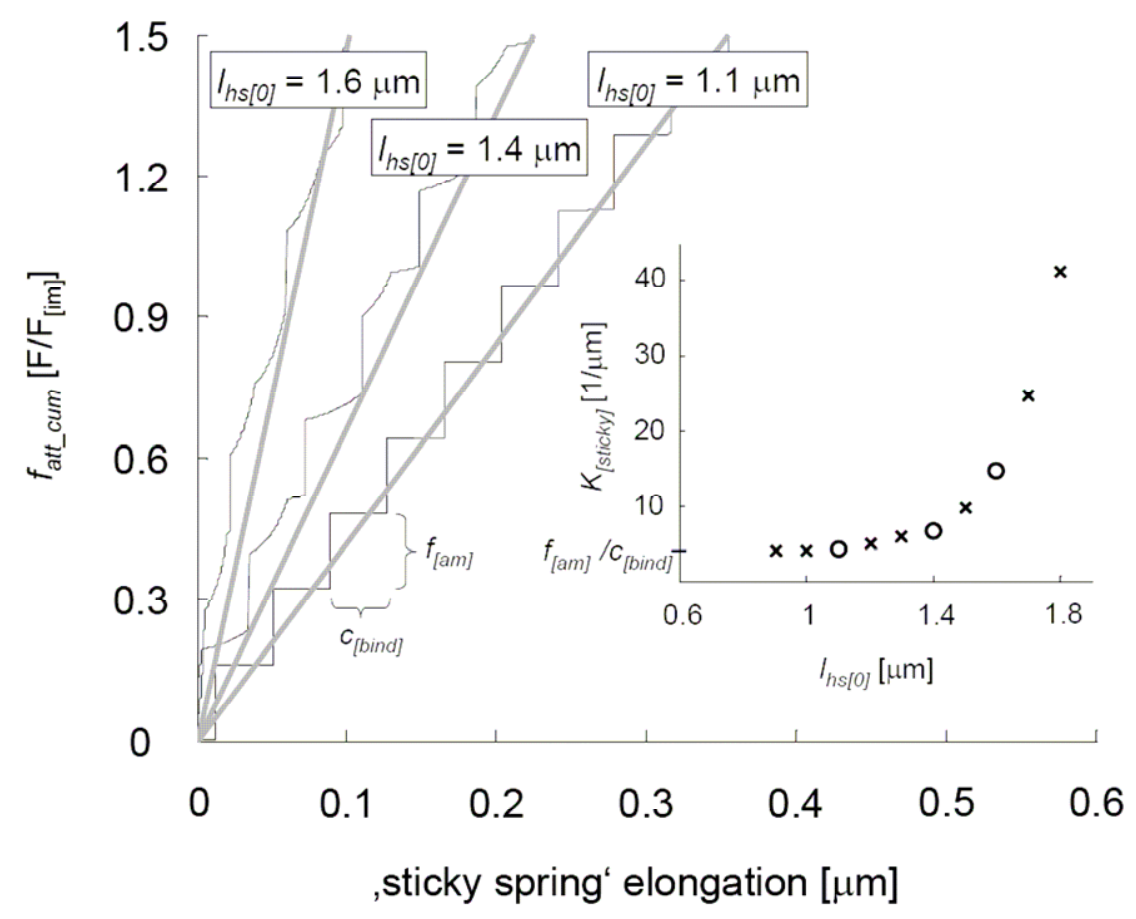

A

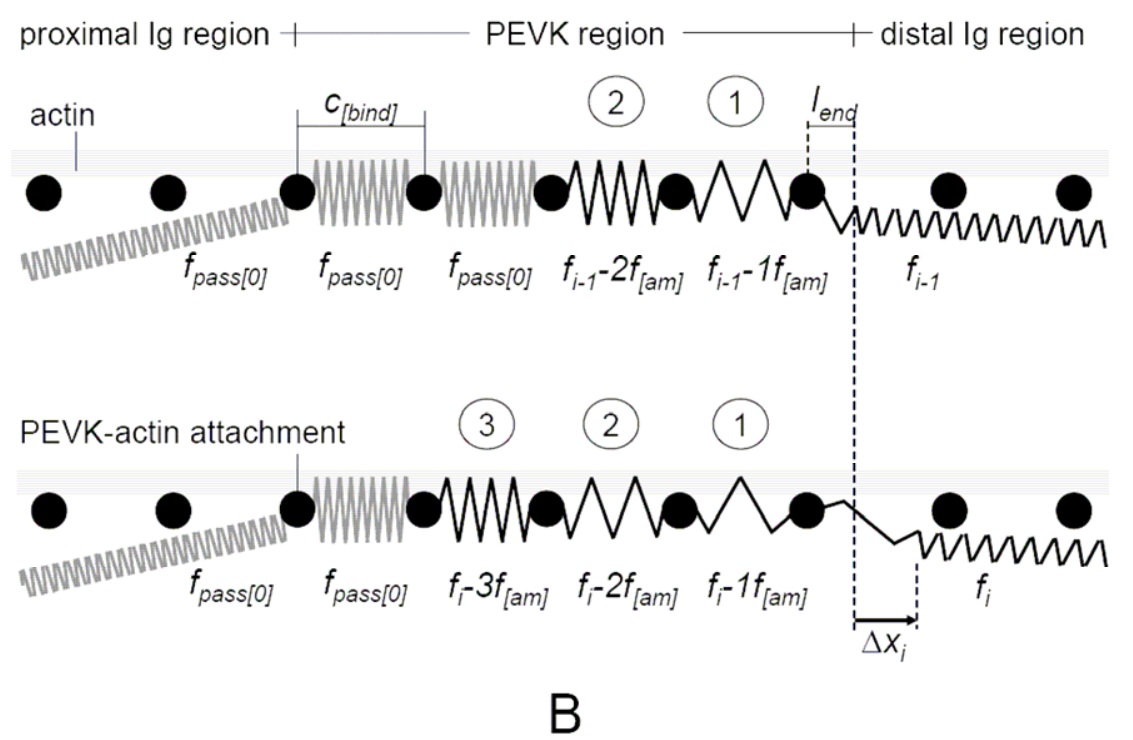

Figure B.1 
780 Fig. C.1. Linear approximation of 'sticky-spring' peel-off force during the second shortening phase. (A)

781 Discontinuous peel-off force dependent on selected initial half-sarcomere lengths $l_{h s[0]}$ plotted vs. second-

782 phase half-sarcomere shortening $l_{h s 1}-l_{h s}$. The corresponding approximate linear stiffness $k_{[\text {short_2] }}$

783 depending on $l_{h s[0]}$ is shown in the inset (circles). The constant distance from peak to peak $\Delta l_{[p e a k]}$ is

784 indicated for shortening from $l_{h s 0}=1.6 \mu \mathrm{m}$. (B) According to the model, the mechanical energy (black

785 lines) expended to peel off the PEVK region changes approximately with the square of the displacement

786 similar to a linear spring. Parabolic approximation (grey lines) yields $\left.k_{[s h o r t} 2\right]$.

A

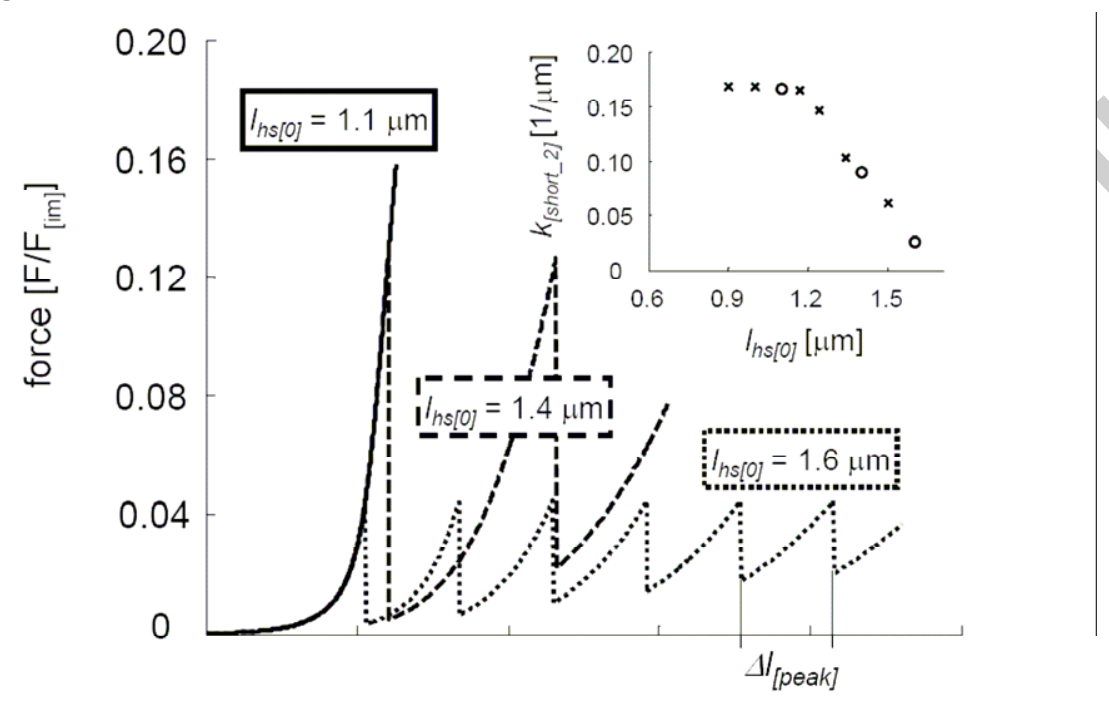

B

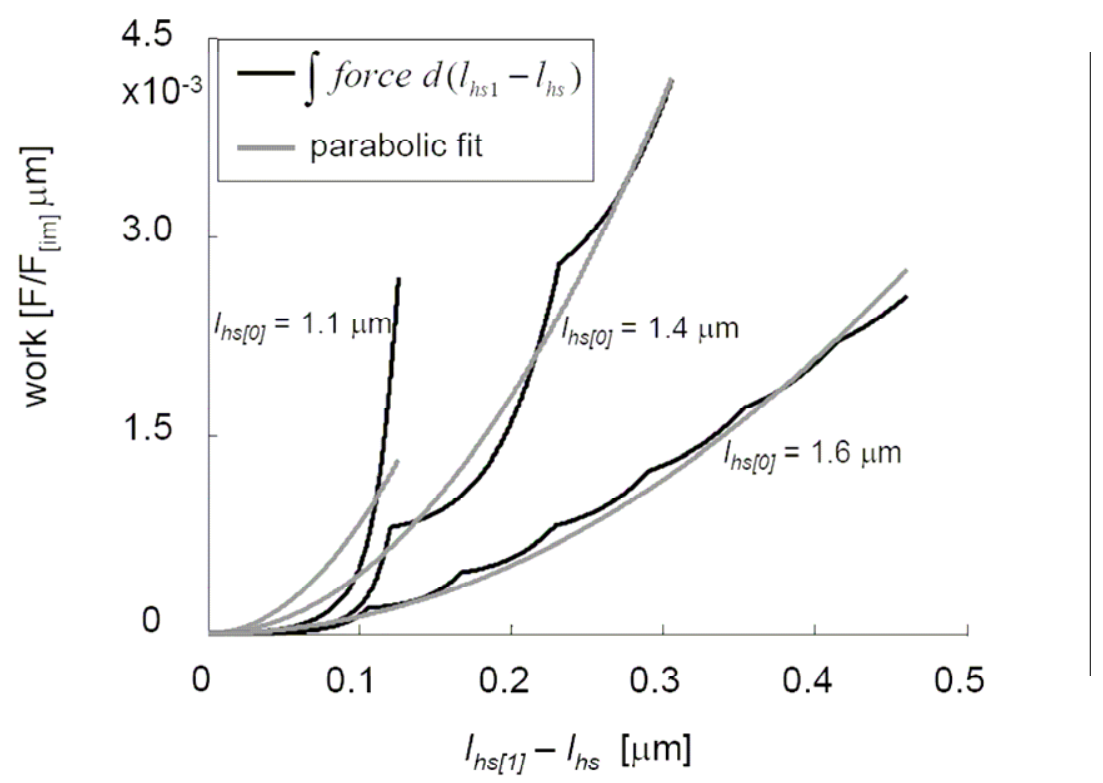

